\title{
Mechanically Reconfigurable Multi-Functional Meta-Optics
}

\author{
Conner Ballew, Gregory Roberts, Philip Camayd-Muñoz, Maximilien F. Debbas, and Andrei Faraon* \\ Kavli Nanoscience Institute and Thomas J. Watson Sr. Laboratory of Applied Physics, California Institute of Technology, Pasadena, California \\ 91125, USA \\ *faraon@caltech.edu
}

\begin{abstract}
We explore mechanically reconfigurable meta-optics with switchable optical functionality. 3D-printed microwave devices featuring broadband focusing, spectral demultiplexing, and polarization splitting are measured with various reconfiguration schemes, including shearing and auxetic transformations. $\odot 2021$ The Author(s)
\end{abstract}

Optical elements have undergone substantial miniaturization over the past two decades, enabled by the improvement of nanofabrication techniques that can pattern subwavelength features in dielectric materials. Dielectric devices with subwavelength thickness and subwavelength etched features, known as metasurfaces, can mimic the optical functionality of bulk components with drastically reduced thickness [1]. Furthermore, recent demonstrations have shown that metasurfaces can synthesize the function of multiple bulk optical elements into a single thin layer [2]. Examples include polarization control, spectral filters, and arbitrary phase holograms [3]. However, the performance of metasurfaces is limited by the number of modes, or optical degrees of freedom, that the device can support $[4,5]$. The number of modes scales with the refractive index contrast and the volume of the device, so adding thickness to the metasurface can improve performance.

Intuitively the size of the system can be increased by introducing a cascaded system of metasurfaces. Such systems have encouraged new technologies, such as a "folded" metasurface-based hyper-spectrometer [6], metasurface-based lens doublets [7], and retroreflectors [8]. Yet the system size is dictated by the need to spatially separate metasurfaces to preserve their prescribed functionality - the local phase approximation employed in metasurface design breaks down when multiple scattering events occur in the device region. A continuous volume of 3D-patterned material can be used to eliminate free-space propagation between patterned elements. Adjoint-based inverse-design techniques have been demonstrated to efficiently search the large solution space of 3D structures for optimal solutions, while respecting fabrication constraints. Recent examples include spectral demultiplexers on both integrated waveguide and free-space platforms $[9,10]$.

In this work we use mechanical reconfiguration to expand the multifunctional capabilities of dielectric metaoptics. Mechanical reconfiguration schemes are coupled with an adjoint-based topology optimization algorithm to find locally optimal refractive index distributions for performing a variety of optical functions, including spectral demultiplexing, broadband focusing, and polarization splitting across a bandwidth similar to the bandwidth of visible light $(450 \mathrm{~nm}-690 \mathrm{~nm})$. The optimization process consists of retrieving the gradient of the various figures of merits with respect to device permittivity using the adjoint method, then adjusting the device pixels proportionally to the gradient until performance is maximized. During the optimization the device is modelled as a fictitious greyscale permittivity between the two material boundaries. As the optimization proceeds, the pixels tend to be pushed towards the material boundaries. The optimization is terminated when device performance is satisfactory, and the device permittivity is rounded to the nearest material permittivity. At this point, any performance lost during this rounding operation can be recovered with a level-set optimization as discussed in Ref. [11]. However, this technique was not used in this work since the device performance was not greatly reduced by this operation.

The first device uses an auxetic transformation, meaning it exhibits a negative Poisson's ratio, to switch between broadband focusing and spectral demultiplexing [12]. The auxetic device consists of rotatable rigid squares, connected at appropriate hinge points as shown in Fig. 1a. The broadband focusing configuration focuses a normally incident TE planewave to the center pixel, as shown in Fig. 1b. The spectral demultiplexing configuration, shown in Fig. 1c, focuses the 7.6-8.9 GHz band to the left pixel, the 8.9-10.2 GHz band to the center pixel, and the 10.2-11.6 $\mathrm{GHz}$ band to the right pixel. This functionality is conceptually similar to color-sensing on an image-sensor array, but with much greater efficiency than traditional absorptive-based color sensors. This device is experimentally verified at microwave frequencies spanning $7.6 \mathrm{GHz}$ to $11.6 \mathrm{GHz}$, using a material system consisting of air and 3D-printed polylactide acid (PLA). The device is measured in a home-built anechoic chamber that supports only the fundamental TEM mode. The field profiles are integrated over the frequency band of interest for each figure of merit and compared with simulations. The results are shown in Fig. 1(d-g).

The second device is actuated by shearing alternating layers with respect to each other, similar to MEMSbased electrostatic actuation. This device also features broadband focusing (Fig. 1i) and spectral demultiplexing (Fig. 1h), but adds polarization splitting as a third functionality (Fig. 1j), focusing the TE input to the left pixel and 
the TM input to the center pixel. Furthermore, each functionality considers both TE and TM polarizations. It was found that this demanding multifunctionality required the device be approximately $3 \times$ thicker than the auxetic device to obtain similar efficiencies for each functionality. The net shear between each layer is $6.2 \mathrm{~mm}$, which is approximately two wavelengths, demonstrating an impressive switching of optical functionality with a wavelengthscale footprint and actuation distance. This device was not experimentally corroborated since the microwave test setup cannot support TM modes.

The ideas explored in this work offer exciting prospects for metaoptics moving forward. In particular, the devices studied here could be 3D-printed at the micro- or nanoscale and combined with appropriate actuation mechanisms to enable near-infrared and visible devices. Furthermore, such devices could be tiled over imaging arrays or digital micromirror devices to enable new camera and display technologies.

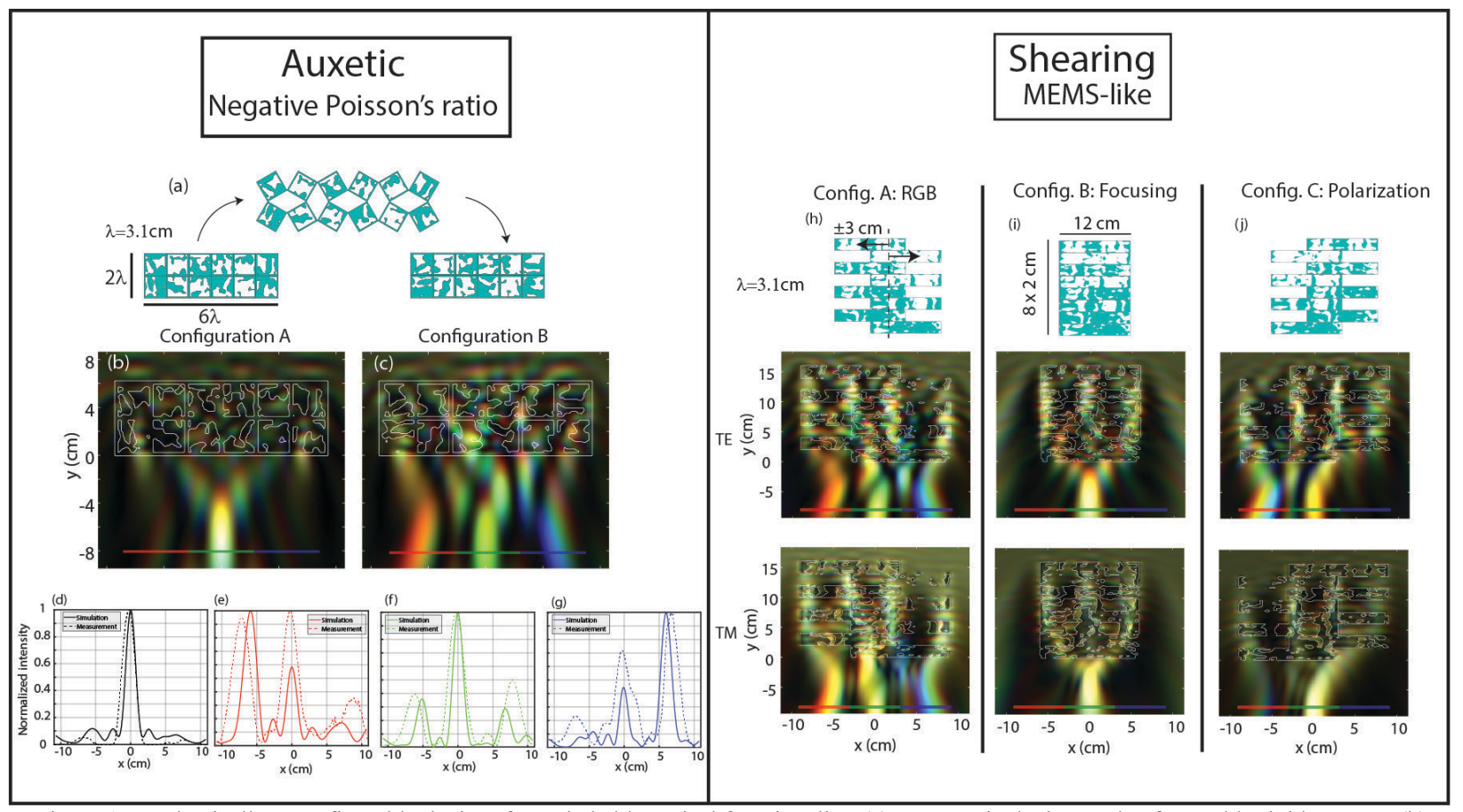

Figure 1: Mechanically reconfigurable devices for switchable optical functionality. (a) An auxetic device made of rotatable rigid squares. (b) Broadband focusing configuration. (c) Spectral demultiplexing configuration. (d-g) Normalized intensity profiles integrated over (d) 7.6-11.6 GHz in Config. A, (e) 7.6-8.9 GHz in Config. B, (f) 8.9-10.2 GHz in Config. B, (g) 10.2-11.6 GHz in Config. B. (h-j) The shearing-based device in its (h) spectral demultiplexing config., (i) broadband focusing config., and (j) polarization-splitting config. All 2D plots are drawn by scaling the wavelengths by a factor of $\times 59,618$ such that they correspond to visible wavelengths and are converted to the appropriate hue using a CIE 1931 color mapping.

\section{References}

1. P. Lalanne, S. Astilean, P. Chavel, E. Cambril, and H. Launois, "Blazed binary subwavelength gratings with efficiencies larger than those of conventional échelette gratings," Opt. Lett. 23(14), 1081 (1998).

2. $\quad$ N. Yu and F. Capasso, "Flat optics with designer metasurfaces," Nat. Mater. 13(2), 139-150 (2014).

3. A. Arbabi, Y. Horie, M. Bagheri, and A. Faraon, "Dielectric metasurfaces for complete control of phase and polarization with subwavelength spatial resolution and high transmission," Nat. Nanotechnol. 10(11), 937-943 (2015).

4. $\quad$ D. A. B. Miller, "Fundamental limit for optical components," J. Opt. Soc. Am. B 24(10), A1 (2007).

5. $\quad$ A. Arbabi and A. Faraon, "Fundamental limits of ultrathin metasurfaces," Sci. Rep. 7(1), 1-9 (2017).

6. M. Faraji-Dana, E. Arbabi, H. Kwon, S. M. Kamali, A. Arbabi, J. G. Bartholomew, and A. Faraon, "Hyperspectral Imager with Folded Metasurface Optics," ACS Photonics 6(8), 2161-2167 (2019).

7. $\quad$ B. Groever, W. T. Chen, and F. Capasso, "Meta-lens doublet in the visible region," Nano Lett. 17(8), $4902-4907$ (2017).

8. A. Arbabi, E. Arbabi, Y. Horie, S. M. Kamali, and A. Faraon, "Planar metasurface retroreflector," Nat. Photonics 11(7), 415-420 (2017).

9. A. Y. Piggott, J. Lu, K. G. Lagoudakis, J. Petykiewicz, T. M. Babinec, and J. Vucković, "Inverse design and demonstration of a compact and broadband on-chip wavelength demultiplexer," Nat. Photonics 9(6), 374-377 (2015).

10. P. Camayd-Muñoz, C. Ballew, G. Roberts, and A. Faraon, "Multifunctional volumetric meta-optics for color and polarization image sensors," Optica 7(4), 280 (2020).

11. L. Su, D. Vercruysse, J. Skarda, N. V. Sapra, J. A. Petykiewicz, and J. Vučković, "Nanophotonic inverse design with SPINS: Software architecture and practical considerations," Appl. Phys. Rev. 7(1), 011407 (2020).

12. J. N. Grima and K. E. Evans, "Auxetic behavior from rotating squares," J. Mater. Sci. Lett. 19(17), 1563-1565 (2000). 